\title{
A case report of anti- $\mathrm{N}$-methyl-d-aspartate receptor autoimmune encephalitis with sensory attack. Is limbic encephalitis only "limbic"?
}

\author{
Sheng Chen ${ }^{1 *}$, Xiao-Jie Zhang ${ }^{2 *}$, Meng-Sha Yao1, Xing-Hua Luan ${ }^{1}$, Fei Yuan ${ }^{3}$, Jun Liu', Shu-Feng Chen ${ }^{1}$, \\ Chen-Fei Jia ${ }^{4}$, Sheng-Di Chen ${ }^{1}$
}

${ }^{I}$ Department of Neurology, Ruijin Hospital, Shanghai Jiaotong University School of Medicine, Shanghai 200025, China.

${ }^{2}$ Department of Neurology, Shanghai Sixth People's Hospital, Shanghai Jiaotong University School of Medicine, Shanghai 200025, China.

${ }^{3}$ Department of Pathology, Ruijin Hospital, Shanghai Jiaotong University School of Medicine, Shanghai 200025, China.

${ }^{4}$ Department of Anesthesiology, Ruijin Hospital, Shanghai Jiaotong University School of Medicine, Shanghai 200025, China.

"The first two authors contributed equally to this work.

Correspondence to: Dr. Sheng-Di Chen, Department of Neurology, Ruijin Hospital, Shanghai Jiaotong University School of Medicine, Shanghai 200025, China. E-mail: chen_sd@medmail.com.cn

How to cite this article: Chen S, Zhang XJ, Yao MS, Luan XH, Yuan F, Liu J, Chen SF, Jia CF, Chen SD. A case report of anti- $N$-methyl-d-aspartate receptor autoimmune encephalitis with sensory attack. Is limbic encephalitis only "limbic"? Neuroimmunol Neuroinflammation 2017;4:78-81.

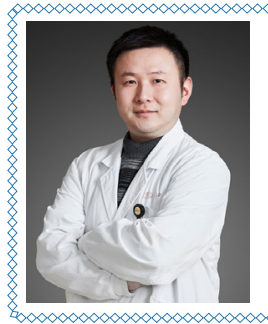

Dr. Sheng Chen is an attending physician, also American ECFMG Certificated Physician, and works in Department of Neurology, Ruijin Hospital, Shanghai Jiaotong University School of Medicine. He is a member of American and Chinese Society of Neuroscience and Neurology. His main research focus is neuro-immunology and neurodegenerative diseases. He had published more than $30 \mathrm{SCl}$ articles including in Journal of Autophagy and Brain Pathology. He also acquired the support of both National Natural Sciences Foundation of China and Shanghai New Youth Science and Technology Project.

Article history:

Received: 10-08-2016

Accepted: 28-12-2016

Published: 10-05-2017

Key words:

Limbic encephalitis,

anti- $N$-methyl-d-aspartate-

receptor encephalitis,

antibody

\section{ABSTRACT}

To emphasize the early diagnosis and treatment of anti- $N$-methyl-d-aspartate-receptor (NMDAR) autoimmune encephalitis, a rare clinical condition, teratoma-related, anti-NMDAR encephalitis should be suspected if young patients present with psychiatric, movement, and sensory symptoms. Early diagnosis and treatment can decrease the mortality and disability rate.

cc) (i) (2) This is an open access article distributed under the terms of the Creative Commons AttributioncC NG SA NonCommercial-ShareAlike 3.0 License, which allows others to remix, tweak, and build upon the work non-commercially, as long as the author is credited and the new creations are licensed under the identical terms. 


\section{INTRODUCTION}

Limbic encephalitis is believed to be a disorder affecting the medial temporal lobe of the brain. The underlying cause can be either of autoimmune origin or viral infection. $\mathrm{N}$-methyl-d-aspartate-receptor (NMDAR) antibodies, voltage-gated potassium channel antibodies, and glutamic acid decarboxylase receptor antibodies are the most common causes of autoimmune limbic encephalitis. Although considered as limbic encephalitis, however, these diseases are not restricted to the limbic system. Here, we present a case of anti-NMDAR encephalitis with teratoma. This patient showed not only "limbic symptoms" but also sensory disturbances and extrapyramidal symptoms, which suggested that more extensive lesions, including cortical and subcortical regions, might have been involved.

\section{CASE REPORT}

A 15-year-old Chinese female presented with a 1 month worsening of mania and memory problems. She also reported mild fever, generalized fatigue, anorexia, right hand abnormal involuntary movement, and paresthenia on the right side of her body. She denied any infectious history before symptoms started. She also denied any headache, vertigo, nausea, or blurry vision. Neurological examination showed an irritable patient with impaired short-term memory, echolalia, echoapraxia, stereotype movement in her right hand, and oral-facial dyskinesias.

On admission, lumbar puncture found 8 white blood cells (per $\mathrm{mm}^{3}$ ), glucose $40 \mathrm{mg} / \mathrm{dL}$, and protein $40 \mathrm{mg} / \mathrm{dL}$. Serum human immunodeficiency virus antibody, syphilis rapid plasma reagin, antinuclear antibody, antibodies to extractable nuclear antigens, antineutrophil cytoplasmic antibody, anti-double stranded DNA, thyroid peroxidase antibody, and the venereal disease research laboratory test were negative. Electroencephalography (EEG) showed slowing of the normal background frequency. Cranial magnetic resonance imaging (MRI) showed no obvious abnormal signal changes, including in the limbic system. Viral encephalitis was first suspected, therefore intravenous high doses of steroids, acyclovir, and glycerol were administered. After treatment initiation, the patient's symptoms deteriorated, with worsening consciousness, intermittent and alternating mania attacks, abulia, echolalia, echoapraxia, 2 episodes of generalized seizures, and persistent right side paresthenia. Serum anti-neuromyelitis optica (NMO)/aquaporin-4 (AQP4) antibody was negative. Blood gas failed to show any hypoventilation.
Repeated lumbar puncture showed no significant change in cell count, glucose, or protein level. Herpes simplex virus (HSV) and cytomegalovirus (CMV) polymerase chain reactions were negative in cerebrospinal fluid (CSF). The immunoglobulin G ( $\mathrm{IgG}$ ) index was 0.5 . CSF oligoclonal banding was also negative. Repeated $3 T$ MRI also failed to show any positive findings [Figure $1 \mathrm{~A}$ ]. An fluorodeoxyglucose (FDG)-positron emission tomography (PET) scan indicated hypo-metabolism in the right temporal and bilateral occipital lobes [Figure 1B]. NMDAR antibody testing was performed using a standardized laboratory assay..$^{[1]}$ The result revealed positive anti-NMDAR antibodies both in serum and CSF [Figure 1G]. Abdominal computed tomography, with $5 \mathrm{~mm}$ slice thickness, and pelvic MRI were performed and revealed a $5.1 \mathrm{~cm} \times 7 \mathrm{~cm}$ fat intensity cystic lesion in the rectouterine pouch, which indicated the possibility of a teratoma [Figure $1 \mathrm{C}$ and D]. Teratomaassociated anti-NMDA receptor encephalitis was then suspected, and intravenous immunoglobulin (IVIG) $(0.4 \mathrm{~g} / \mathrm{kg}$ per day) was administrated for 5 days. Valproic acid was used to control seizure attacks. The patient's symptoms improved 7 days after IVIG infusion, which was characterized by memory and consciousness improvement. Twenty days after admission to our hospital, the patient underwent a laparoscopic operation for complete teratoma resection [Figure $1 \mathrm{E}$ and $\mathrm{F}$ ]. Immunochemistry staining revealed a $4.8 \mathrm{~cm} \times 6.9 \mathrm{~cm}$ cystic tumor with fat, hair, teeth, and brain tissue components. The pathological diagnosis was mature cystic teratoma containing brain tissue. Using immunochemistry staining, we found that the brain tissue contained NMDA NR1/NR2 subunit receptor positive neurons [Figure 1H]. Patient CSF samples were screened for NMDAR IgG antibodies by immunofluorescence using human embryonic kidney (HEK) 293 cells transfected with the NR1 subunit of the NMDAR complex (Euroimmun, Germany) [Figure 1G]. Nontransfected HEK 293 cells served as a negative control for nonspecific fluorescence [Figure $1 \mathrm{I}$ and J].

The patient was discharged 1 month after admission. At that time, her symptoms significantly improved. EEG monitoring was performed 2 months after discharge, which revealed a normal pattern. Valproic acid was gradually tapered down. Six months after discharge, this patient was free of all medications. Her cognitive function was fully recovered and her psychiatric symptom, involuntary movement, sensory disturbance, and oral-facial dyskinesias disappeared. Cranial MRI was repeated 1 year after discharge, and no brain atrophy was observed. 


\section{DISCUSSION}

Anti-NMDAR encephalitis is a type of limbic encephalitis that is typically found in young women with teratomas. ${ }^{[2]}$ This kind of encephalitis is usually subacute at onset with significant psychiatric symptoms, including agitation, mania, hallucination, aggression as well as cognitive dysfunction. ${ }^{[3]}$ Some patients will develop echolalia, echoapraxia, involuntary movements, such as stereotype, central hypoventilation, and autonomic instability, which have been considered more specific characteristics for helping in diagnosis. ${ }^{[4]}$ Although extreme delta brush on an EEG can be another specific diagnostic marker, most patients, including
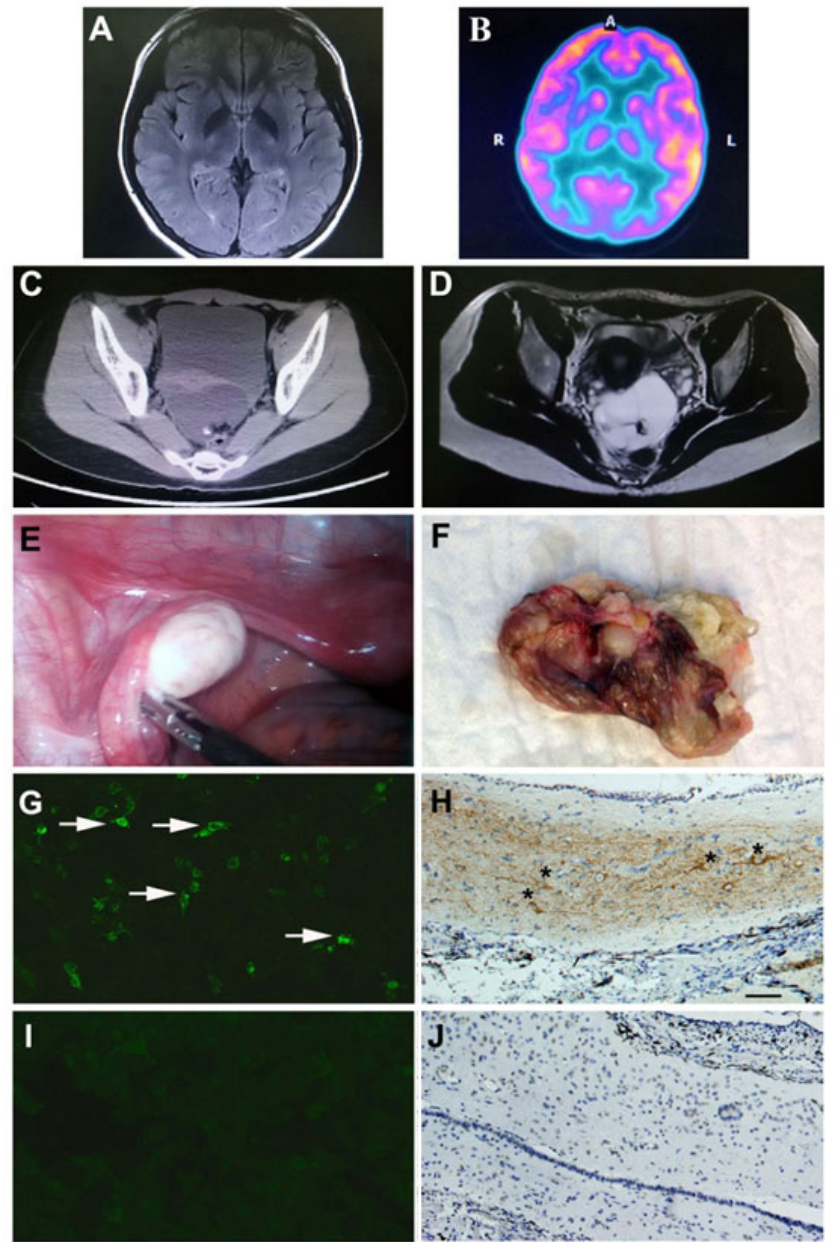

Figure 1: (A) Normal cranial MRI scan; (B) FDG-PET scan showed hypo-metabolism in the right temporal and bilateral occipital lobes; ( $C$ and $D$ ) pelvic computed tomography and MRI revealed a cystic lesion; ( $E$ and $F$ ) ovarian teratoma was resected during laparoscopy. The tumor consisted of bone, teeth and hair; (G) positive HEK 293 cells with anti-NMDAR antibodies using patient's cerebrospinal fluid (white arrows indicate the positive cells); $(\mathrm{H})$ positive immunostaining of teratoma using NMDA NR1 receptor antibody (scale bar $=100 \mu \mathrm{m}$, black asterisks indicate the NR1 positive cells). The negative control of NMDAR immunostaining in HEK 293 cells (I) and in teratoma tissue (J). MRI: magnetic resonance imaging; NMDAR: $N$-methyl-d-aspartate-receptor; HEK: human embryonic kidney; FDG: fluorodeoxyglucose; PET: positron emission tomography this one, only present with nonspecific background change or diffuse slow waves, especially at the early stage of disease. ${ }^{[5]}$ Usually, bilateral medial temporal lobe signal change on MRI scans raise suspicions for limbic encephalitis. ${ }^{[6]}$ However, normal MRI results cannot exclude the diagnosis. Our patient underwent 3T cranial MRI scans twice, and no obvious change was found, including in the limbic system. It is known that, although NMDARs are more concentrated in the hippocampal area, they also can be found in many other areas of the brain, including sensory and association cortex and subcortical regions. ${ }^{[7]}$ The widespread distribution of the receptor in cortical regions could explain the diffuse slow waves on the EEGs and the persistent sensory symptoms seen in our patient. Oral-facial dyskinesias indicated basal ganglion involvement. Although most anti-NMDAR encephalitis is limbic, some patients may have more extensive lesions, including cortical and subcortical; thus, limbic encephalitis is not always only limbic. Likewise, an FDG-PET scan of our patient showed hypo-metabolism in multiple brain regions. In addition, not all patients have positive MRI findings, especially at the early stage of disease, and we speculated that MRI scanning may not always be reliable for early diagnosis and differentiation. The differential diagnosis of antiNMDAR encephalitis, excluded HSV encephalitis, CMV encephalitis, Hashimoto's encephalopathy, systemic lupus erythematosus encephalopathy, antiphospholipid antibody syndrome, Sjögren's syndrome, and primary central nervous angiitis. ${ }^{\left[{ }^{[8]}\right.}$ We also tested for anti-AQP4 to exclude its co-occurrence with anti-NMDAR. ${ }^{[9,10]}$ This patient was steroid unresponsive, since a high dose of intravenous administration of steroids failed to improve her symptoms. After IVIG infusion and tumor resection, she recovered to normal status in a short period of time, and we gradually tapered down all her medications. This patient did not show any relapse 1 year after discharge. Although most studies indicated recovery was a slow process for anti-NMDAR encephalitis, our experience in patient with teratoma and receiving tumor resection, had good prognosis and fast recovery time. In addition, these patients are not suggested to continue long-time immunosuppressant.

Anti-NMDAR encephalitis is a rare clinical condition and may associate with ovarian teratoma. This kind of autoimmune limbic encephalitis may extend to cortical and subcortical regions. Cranial MRI is not reliable for early diagnosis. Patients with teratoma usually have good prognosis after mass resection.

\section{Authors' contributions}

Conception, diagnosis and design: S. Chen

Manuscript preparation: X.J. Zhang 
Data collection and assembly of data: M.S. Yao, X.H. Luan

Pathology diagnosis: F. Yuan

Manuscript revision: J. Liu, S.F. Chen, C.F. Jia

Final approval of manuscript: S.D. Chen

\section{Financial support and sponsorship}

This work was supported by grants from the National Natural Sciences Foundation of China (81671241); Shanghai New Youth Science and Technology Project (15QA1403000); Shanghai Youth Doctor Training Programme and Shanghai Jiaotong University Medicine Engineering Cross Programme (YG2014MS54).

\section{Conflicts of interest}

There are no conflicts of interest.

\section{Patient consent}

Patient consent was obtained from the patient.

\section{Ethics approval}

Data collection in our study involving the patient is consistent with the ethical standards of the institution's ethics committee.

\section{REFERENCES}

1. Frechette ES, Zhou L, Galetta SL, Chen L, Dalmau J. Prolonged follow-up and CSF antibody titers in a patient with anti-NMDA receptor encephalitis. Neurology 2011;76:S64-6.

2. Mann AP, Grebenciucova E, Lukas RV. Anti-N-methyl-D-aspartatereceptor encephalitis: diagnosis, optimal management, and challenges. Ther Clin Risk Manag 2014;10:517-25.

3. Mann A, Machado NM, Liu N, Mazin AH, Silver K, Afzal KI A multidisciplinary approach to the treatment of anti-NMDAreceptor antibody encephalitis: a case and review of the literature. $J$ Neuropsychiatry Clin Neurosci 2012;24:247-54.

4. Goldberg EM, Titulaer M, de Blank PM, Sievert A, Ryan N. Anti$\mathrm{N}$-methyl-D-aspartate receptor-mediated encephalitis in infants and toddlers: case report and review of the literature. Pediatr Neurol 2014;50:181-4.

5. VanHaerents S, Stillman A, Inoa V, Searls DE, Herman ST. Early and persistent extreme delta brush in a patient with anti-NMDA receptor encephalitis. Epilepsy Behav Case Rep 2014;2:67-70.

6. Mittal MK, Rabinstein AA, Hocker SE, Pittock SJ, M Wijdicks EF, McKeon A. Autoimmune encephalitis in the ICU: analysis of phenotypes, serologic findings, and outcomes. Neurocrit Care 2016;24:240-50

7. Ghasemi M, Phillips C, Trillo L, De Miguel Z, Das D, Salehi A. The role of NMDA receptors in the pathophysiology and treatment of mood disorders. Neurosci Biobehav Rev 2014;47:336-58.

8. Barry H, Byrne S, Barrett E, Murphy KC, Cotter DR. Anti-N-methyld-aspartate receptor encephalitis: review of clinical presentation, diagnosis and treatment. BJPsych Bull 2015;39:19-23.

9. Hacohen Y, Absoud M, Hemingway C, Jacobson L, Lin JP, Pike M, Pullaperuma S, Siddiqui A, Wassmer E, Waters P, Irani SR, Buckley C, Vincent A, Lim M. NMDA receptor antibodies associated with distinct white matter syndromes. Neurol Neuroimmunol Neuroinflamm 2014;1:e2

10. Orengo JP, Pekmezci M, Cree BA. Simultaneous serum aquaporin-4 antibody and CSF NMDA receptor antibody-positive encephalitis. Neurol Neuroimmunol Neuroinflamm 2015;2:e101. 